\title{
Reversible Electrical Reduction and Oxidation of Graphene Oxide
}

\author{
Okan Öner Ekiz, ${ }^{*}$ Mustafa Urel, Hasan Güner, Alpay Koray Mizrak, and Aykutlu Dâna* \\ UNAM-Institute of Materials Science and Nanotechnology, Bilkent University, 06800 Ankara, Turkey
}

G raphene ${ }^{1-3}$ has received tremendous attention in recent years due to its potential applications in electronics, ${ }^{4-6}$ sensors, ${ }^{7-9}$ energy storage, ${ }^{10-12}$ and optics. ${ }^{13-15}$ Despite the extreme intrinsic mobility of graphene, ${ }^{16}$ semimetallic electronic band structure limited its use in high-performance transistors. Quantum confinement effects were theoretically ${ }^{17,42}$ and experimentally ${ }^{18,19}$ shown to modify the band structure, making graphene a more versatile material. Chemical modification is still being actively sought to form semiconductors in the graphene family and implement conventional optoelectronic device structures. Novel devices have been reported that combine the superior electronic properties of graphene with other chemical and mechanical phenomena, such as resistive switching and electromechanical memories. $^{20-22}$ The mechanism of observed resistive switching in some graphene-based devices remained unclear.

Graphene oxide (GO) is an insulator with a large effective band gap and band structure that depends on the stoichiometry. ${ }^{23,24}$ It has been previously observed that graphene oxide can be reduced controllably at low temperature (low- $T$ ) in ambient atmosphere. ${ }^{25}$ In such a low-temperature reduction process, the resistivity of the films can be continuously monitored and can be used to control the degree of reduction. Interruption of thermal reduction results in partially reduced graphene oxide (PRGO). Conduction mechanisms of such PRGO films are known to be different than multilayer graphene (MLG) films, as evidenced by temperature dependence of resistivity. ${ }^{25-27} \mathrm{~A}$ hopping transport mechanism was thought to be dominant in such films, and limited evidence for the presence of oxygen-rich domains was provided through scanning tunneling microscopy (STM) imaging of PRGO layers. ${ }^{28}$ Recently, thermal site-selective reduction of graphene oxide is demonstrated using a heated atomic force microscope
ABSTRACT We demonstrate that graphene oxide can be reversibly reduced and oxidized using electrical stimulus. Controlled reduction and oxidation in two-terminal devices containing multilayer graphene oxide films are shown to result in switching between partially reduced graphene oxide and graphene, a process which modifies the electronic and optical properties. High-resolution tunneling current and electrostatic force imaging reveal that graphene oxide islands are formed on multilayer graphene, turning graphene into a self-assembled heterostructure random nanomesh. Charge storage and resistive switching behavior is observed in two-terminal devices made of multilayer graphene oxide films, correlated with electrochromic effects. Tip-induced reduction and oxidation are also demonstrated. Results are discussed in terms of thermodynamics of oxidation and reduction reactions.

KEYWORDS: graphene - graphene oxide - electrochemistry · electrochromism electrostatic force microscopy · resistive switching · tip-induced oxidation

tip. $^{29}$ STM has also been used to image hydrogen atoms on graphene and to create nanoscale heterostructure patterns by tipinduced hydrogen desorption. ${ }^{30}$ Recently (in an article published after the submission of this work), a conductive atomic force microscopy tip was used to characterize nanoscale surface conductivity of graphene oxide, and tip-induced reduction was shown to be possible. ${ }^{31}$

In this work, we demonstrate electrically induced reversible reduction of graphene oxide, resulting in changes in the electronic and optical properties of graphene-based thin films. It is observed that optical and electronic property changes are correlated with the formation of a graphene/graphene oxide nanomesh.

\section{RESULTS}

We study the electrical reduction of GO in air, using multilayer GO (MLGO) films deposited on metalized glass substrates. The two-terminal devices consist of thin $(\sim 10-50 \mathrm{~nm}) \mathrm{Pd} / \mathrm{Au}$ planar contacts, separated by $0.3-0.6 \mathrm{~mm}$, with a thin multilayer GO film covering both contacts and in between (Figure 1a). Upon application of a bias to the contacts, the positive contact is seen to be oxidized and the negative
*Address correspodence to
ekiz@bilkent.edu.tr,
aykutlu@unam.bilkent.edu.tr.

Received for review June 23, 2010

Published online March 10, 2011 $10.1021 / \mathrm{nn} 1014215$

(c) 2011 American Chemical Society and accepted March 10, 2011. 


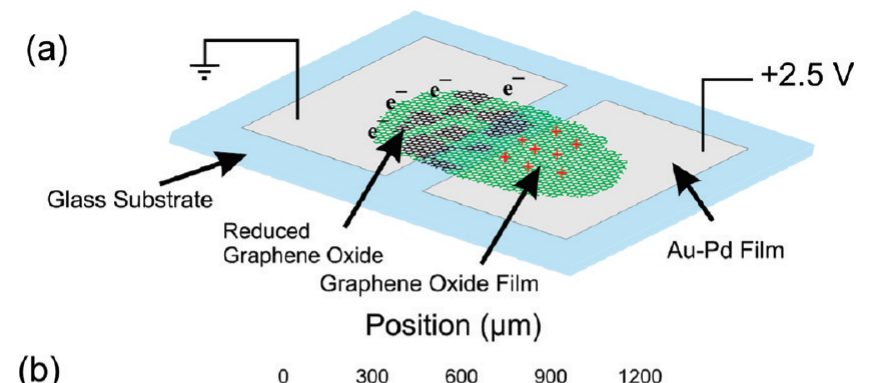

(b)

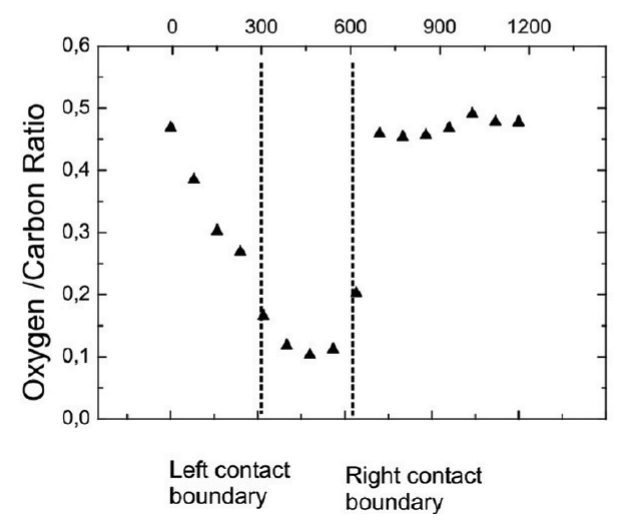

Figure 1. (a) Thin multilayer graphene oxide films (30-60 $\mathrm{nm}$ thickness) can be electrically reduced and oxidized. The twoterminal device is schematically shown. (b) Oxidation and reduction are confirmed by XPS measurements. The oxygen-tocarbon ratio is measured using a $100 \mu \mathrm{m}$ diameter photoelectron collection spot and is plotted as a function of distance across the device. The data show gradual changes in the stoichiometry between the contacts.

contact is seen to be reduced. There is a potential drop between the contacts due to the finite conductivity of the films. The stoichiometry is observed to depend on the distance from the contacts, as confirmed by X-ray photoelectron spectroscopy (XPS) (Figure 1b). The reduction/oxidation can be reversed by reversal of bias. The optical transparencies of the films are also dependent on the oxidation state. Wavelength-dependent linear transmission is measured using a fibercoupled spectrometer near one of the contacts, while the bias is swept quasi-statically between -2.5 and $2.5 \mathrm{~V}$. A gradual increase of the absorption edge as a function of applied bias is observed (Figure 2a). The optical transparency is correlated with the oxidation state (Figure $2 b, c$ ), films being semitransparent in the oxidized state. During cyclic voltage sweeps, it is observed that electrically induced oxidation/reduction takes place with time scales on the order of seconds for applied bias voltages of $\pm 2.5 \mathrm{~V}$. We infer the thickness of the films to be $30-60 \mathrm{~nm}$ using optical measurements, assuming the films have absorption coefficients similar to graphene during the absorbing (reduced) state. $^{32}$

We inspect the surface electronic properties of the two-terminal devices with an atomic force microscope (AFM), imaging the surface topography and conductivity before and after voltage pulses applied between its terminals. Two-terminal devices are subjected to voltage pulses (typically 2 to $3 \mathrm{~V}$ ) while the tip is retracted. After the electrical reduction (or oxidation) process, the device contacts are grounded and the surface conductivity is imaged using a conductive AFM tip, using few millivolt tip bias. It is observed that the surface resistance of MLGO films decreases in the oxidized state, correlated with an increase in the optical transparency of the film (Figure 3a). As the optical microscope of the AFM system did not produce clear images, the changes in the optical transparency of the films are observed with the aid of the optical microscope in transmitted light mode in a separate experiment (Figure 3b). It is observed that the transparent state corresponds to a mostly insulating surface with intermittent conductive domains. Upon application of a reducing pulse, the surface conductivity of the film greatly increases. It is seen that both the number of conductive domains and current per domain increase. When small currents ( $10 \mu \mathrm{A}$ per $\mathrm{mm}$ wide films) are used, the reactions are slow and no significant topographical change is observed in the topography as verified by contact mode AFM images. This suggests that electrical changes are related to the changes in the chemistry of the films and not due to a mechanical reconfiguration. Rapid reduction and oxidation, however, result in delamination of the flakes as evident by increased instability in noncontact AFM images (data not shown). A sequence of optical micrographs shows that oxidation or reduction is not instant and not simultaneously taking place over the film area, but progresses as a front (Figure 4a). This is possibly due to the coupling of the potential distribution inside the film with the film conductivity, which in turn is related to the degree of reduction. It is also observed that, 


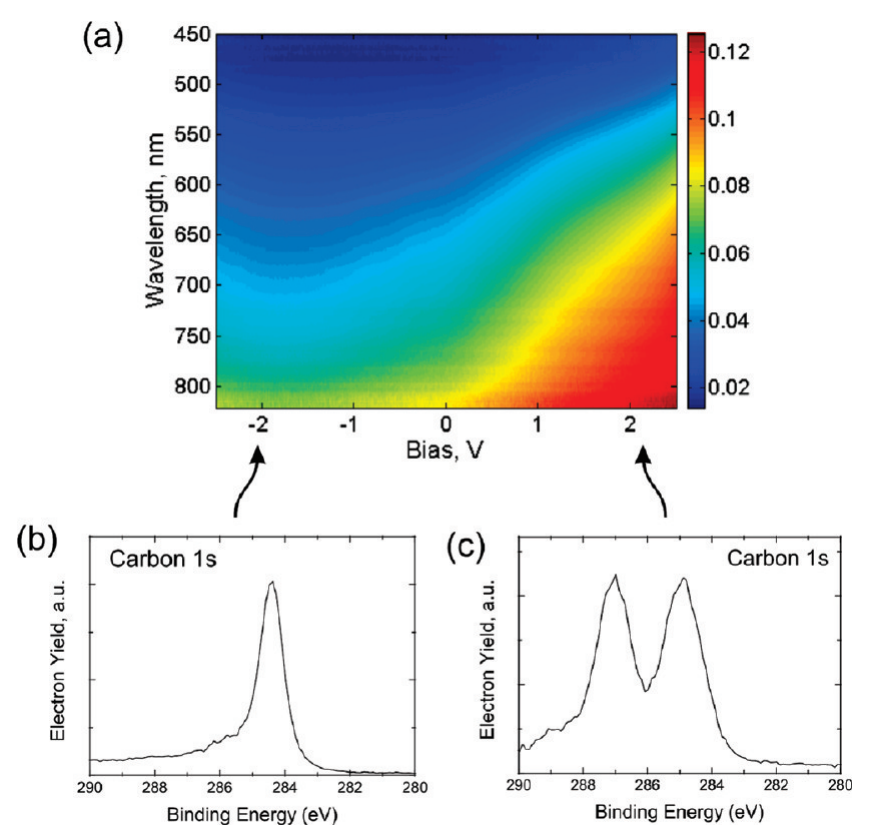

Figure 2. (a) Linear optical transmission of the films is measured using a $25 \mu \mathrm{m}$ core fiber optic light collector near one of the contacts while the bias is swept between -2.5 and $2.5 \mathrm{~V}$. It is observed that wavelength-dependent transmission is adjustable by applying an external bias. Representative XPS spectra that correspond to the (b) reduced and (c) oxidized states show the correlation of optical transparency with stoichiometry.

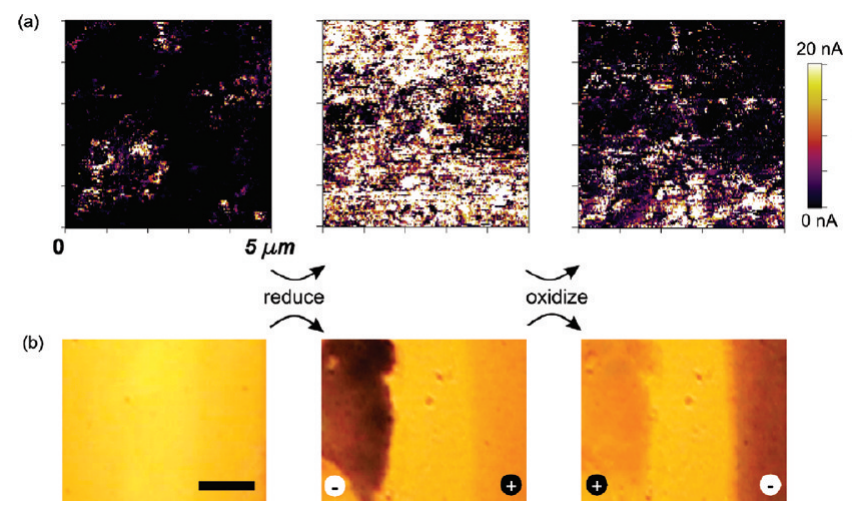

Figure 3. (a) Tunneling current images showing the effect of reversible electrical oxidation and reduction in three consecutive voltage cycles ( $+2 \mathrm{~V},-2 \mathrm{~V},+2 \mathrm{~V}$ for tens of seconds). The images are collected at the same location, by grounding the two contacts and applying a $\sim 10 \mathrm{mV}$ tip bias. The surface conductivity is observed to switch in reduced and oxidized states. (b) Representative optical micrographs (observed by bright-field transmitted light, scale bar $30 \mathrm{um}$ ) of multilayer graphene oxide films show spatially uniform transmission at the beginning of the bias cycles (left), where reduced transmission is observed upon application of bias that reduces the left side of the film (middle, minus sign, dark region). The optical transmission can be manipulated by reversing the polarity of applied bias and causing the left side to oxidize and the right side to reduce (right).

when low $(< \pm 2 \mathrm{~V})$ maximum bias values are used, reversible optical and resistive switching continue for multiple cycles (about 20 in our devices) before the film shows an irreversible reduction in electrical resistance. Our observations suggest that previously observed resistive switching in graphene oxide and graphene devices is a result of chemical modification of graphene due to reversible binding of oxygen. ${ }^{22,23}$ The drop-cast films consist of a large number of flakes. The thickness and conductivity of the films are not very uniform, and we attribute the device failure to formation of highly conductive dendrite-like shorting paths, which are observable in optical images (Figure 4b). As the shorting paths are formed, current paths and voltage distributions inside the film change in a way that the bias needed for the reduction/oxidation process cannot be sustained uniformly and controllably at all parts of the film (see Supporting Information).

We also investigate film surfaces in various oxidation states using high-resolution multifrequency electrostatic force microscopy. Since electrostatic force microscopy is a noncontact and relatively nondestructive measurement technique, we use it as a reliable complementary way of confirming the surface electronic 


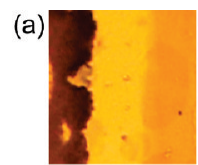

$\mathrm{t}=0 \mathrm{~s}$

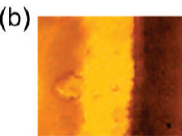

$\mathrm{t}=0 \mathrm{~s}$

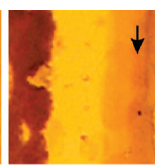

$0.25 \mathrm{~s}$

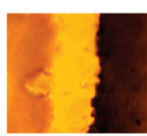

$2.5 \mathrm{~s}$

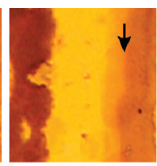

$0.5 \mathrm{~s}$

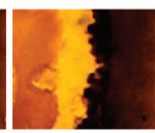

$5 \mathrm{~s}$

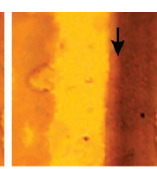

$1 \mathrm{~s}$

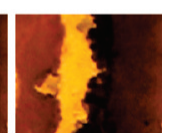

$10 \mathrm{~s}$
Figure 4. (a) Optical micrographs recorded as a function of time show the progress of reduction and oxidation upon sudden reversal of bias polarity. The arrows denote the presence of an advancing reduction front. (b) Failure of the device is observed upon application of a large bias ( 3 to $10 \mathrm{~V}$ for a given device geometry). Failure is due to advancement of dentrite-like conductive (reduced) regions, eventually causing a short in the device.

structure. In the electrostatic force microscopy images, we see that there are interconnected domains of different workfunctions on the PRGO films (Figure 5a, b). Brighter pixels correspond to regions with more negative surface potentials, which we attribute to the presence of bound oxygen and hydroxide. Darker regions correspond to graphene-like channels and quantum dots. Sizes of the graphene dots show a distribution and vary from nanometer to micrometer scale in various states. These observations confirm that partial electrical reduction results in a graphene/graphene oxide heterostructure nanomesh, whose mesh size depends on the degree of reduction. This observation is in parallel with previous theoretical predictions that uniform coverage of graphene oxide is not energetically favorable. ${ }^{23,24}$

Tip-induced electrical oxidation of graphene oxide is demonstrated by using a conductive AFM tip operated in the contact mode. Application of a positive sample bias $(1-2 \mathrm{~V})$ results in oxidation of graphene films, and resulting $\mathrm{GO}$ structures can be observed in the tunneling current image collected with a few millivolt tip bias (Figure 6a). No oxidation is observed for biases under $0.5 \mathrm{~V}$. Reduction of MLGO is observed to occur when the sample is negatively biased. In order to monitor the changes in the electronic properties of the MLGO film surface as a function of bias, the tunneling current is measured between the Pt-coated AFM tip and the MLGO film while the sample potential is swept in a cyclic fashion (Figure 6b). The measurements demonstrate that the flake begins to reduce at relatively small current levels of few tens of picoamperes. Gradual decrease of the band gap during reduction is evidenced by changes in the positive and negative onsets of currents. Before and after the voltage sweeps, surface conductivity is imaged using a small bias (1100 $\mathrm{mV}$ ), and a similar emergent heterostructured mesh of graphene/GO is seen on an individual flake (Figure $6 c$, d). Reduction of the whole flake (or flakes, as highlighted by the red dashed line in Figure $6 \mathrm{~d}$ ) instead of a (a)
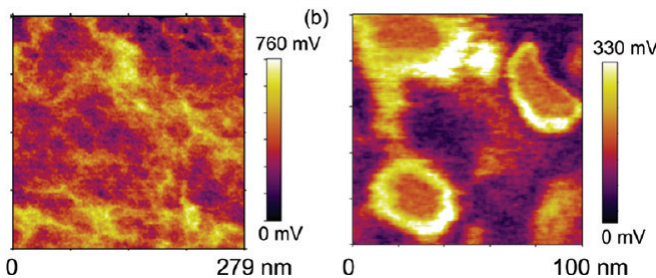

Figure 5. (a) Partially reduced graphene oxide films are investigated by multifrequency electrostatic force microscopy. Regions with different surface potentials are observed, suggesting the presence of a $2 \mathrm{D}$ graphene heterostructure where graphene and graphene oxide domains are simultaneously present. Electrostatic force amplitude is imaged, where bright regions correspond to lower surface potential, or greater oxygen content. (b) Higher resolution electrostatic force map shows that segregated regions with distinct surface potentials are present, with sizes ranging from few nanometers to tens of nanometers.

small spot right below the tip is possibly due to poor grounding of the topmost flake on the insulating MLGO film. The reason for this is that the multilayer graphene oxide films are quite insulating and the effective resistance between the tip and the topmost flake is smaller than the effective resistance between the flake and the ground contact. Therefore, during application of the bias with the tip, whole flake is charged to a potential different than zero. The effective resistance within the flake is also smaller than the resistance between the topmost flake and underlying flakes. Therefore, the carriers injected into the flake diffuse within the flake and facilitate the electrochemical reaction, resulting in reduction of the whole flake. In contrast, tip-induced oxidation is performed on a relatively high conductivity film, and potentials are defined with higher spatial resolution. This is why the rectangle shown in Figure $6 a$ is much better defined in shape than the reduced region of Figure $6 \mathrm{~d}$. The time scales and voltages required for reduction and oxidation are also quite different; oxidation requires longer times and higher voltages. This suggests that the activation energies of reduction and oxidation are different. We assume that oxidation takes place by an electrochemical process within the water meniscus formed between the tip and the sample, similar to tip-induced oxidation of various metals and semiconductors. ${ }^{33-35}$ The observation that reduction takes place at very small current levels suggests that reduction is also an electrochemical process and not a thermal process. Oxygen coverage can be manipulated by applying electric fields in the noncontact mode as well (data not shown), suggesting that the electric field of the tip can also be used to manipulate the surface. Electron injection into graphene oxide is thought to facilitate the reduction, due to weakening of the bonds of negatively charge oxygen atoms with the graphene layer. 
(a)

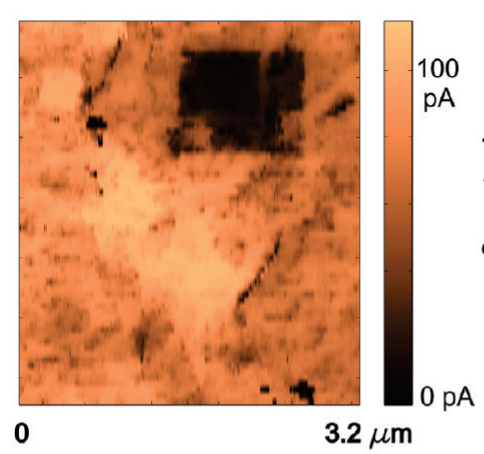

(b)

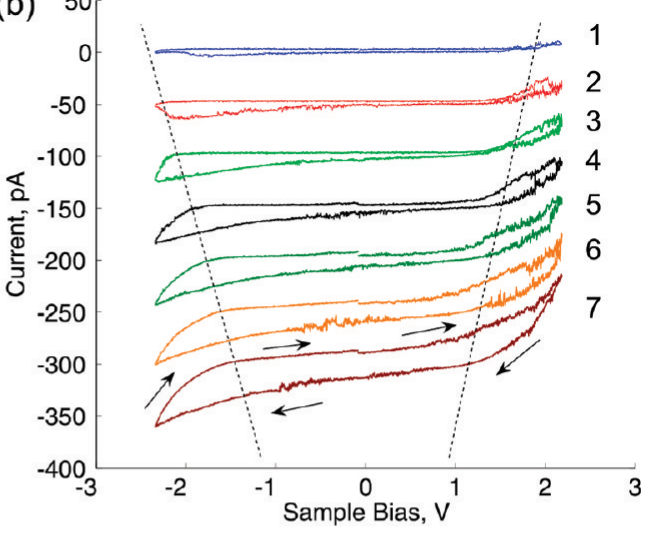

$35 \mathrm{pA}$

(c)

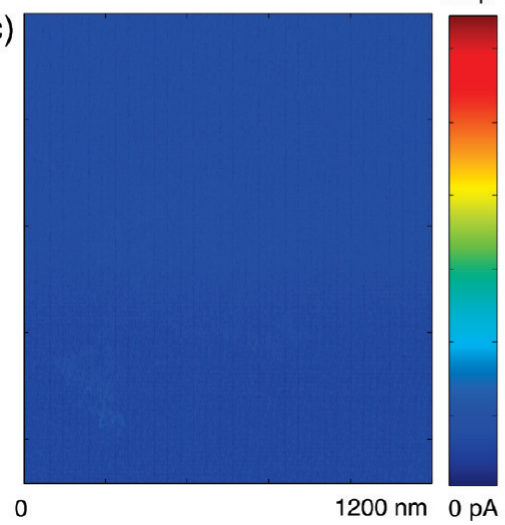

(d)

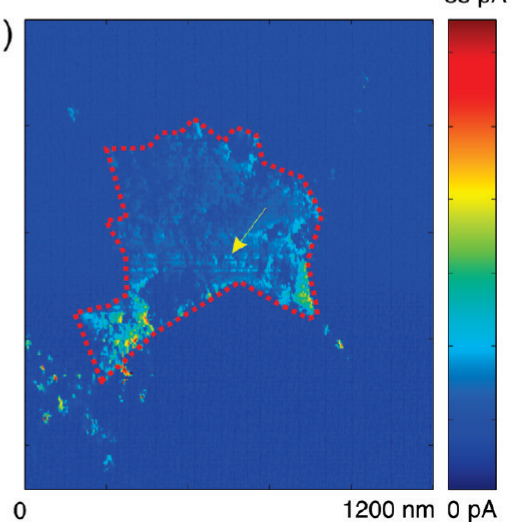

Figure 6. (a) Due to the local electric field induced by a conductive atomic force microscopy tip, graphene can be locally oxidized. (b) Cyclic current versus voltage (IV) measurements are performed on multilayer graphene oxide films on Au/Pd film using a Pt-coated atomic force microscope cantilever. An increase of the current at smaller bias voltage after successive (1 to 7) IV measurements suggests a decrease in the local effective band gap. Despite a symmetric voltage sweep, a net reduction effect is observed, suggesting that oxidation rate is smaller than reduction rate at similar magnitude bias.

(c) Tunneling current map after the first $I V$ measurement shows no or little surface conductivity. (d) Tunneling current map obtained after the 7th $I V$ measurement shows increased current on one flake (or few flakes), suggesting whole individual flakes are reduced. Dashed red contour shows the reduced region and arrow denotes the location of the cantilever tip.

The presence of states that trap electrons inside graphene oxide layers is evidenced by the charge storage effect observed in cyclic current-voltage measurements on two-terminal devices. A graphene oxide device with an apparently insulating surface (Figure 7a) shows nonzero current during a cyclic voltage sweep (Figure 7d). Hysteresis observed in the $I V$ curves demonstrates charge storage (nonzero current at zero bias) and resistive switching (current asymmetry and change of the shape of the forward and backward sweep curves, around $-0.2 \mathrm{~V}$ and $+0.2 \mathrm{~V}$ in Figure $7 \mathrm{~d}$ ). Upon cycling the voltage with increasing maximum voltage levels, reduction takes place, accompanied with resistive switching. Upon further reduction, conductive islands begin to form on the surface similar to those observed for thermal PRGO films (Figure 7b). For the PRGO films, the hysteresis in the current-voltage curves is reduced (Figure 7e). Chemically or thermally reduced multilayer graphene film surfaces do not have conductive dots on the surface (Figure 7c), and they do not display charge storage or resistive switching effects
(Figure 7f). It must be noted that we were not able to observe reversible oxidation when we start with films of chemically reduced graphene. This may be due to the high conductivity of such films and resulting inability to establish significantly differing potentials within the film.

\section{DISCUSSION}

The results show that electrical charge has a strong effect on the thermodynamic equilibrium of graphene/ oxygen system. In ambient atmosphere, positive charge causes oxidation of graphene, and negative charge enhances the reduction of graphene oxide to graphene. Graphene oxide flakes are unstable and slowly decompose to partially reduced graphene oxide. The decomposition process is slow at room temperature. For instance, it is observed that graphene oxide suspensions start to darken in a few months at $25^{\circ} \mathrm{C}$, which indicates the partial reduction of graphene oxide. However, negative charging effectively increases the free energy change ( $\Delta G$ of the decomposition 
(a)

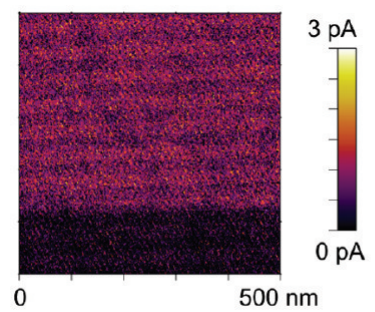

(b)

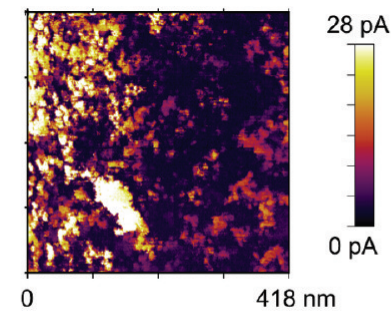

(c)

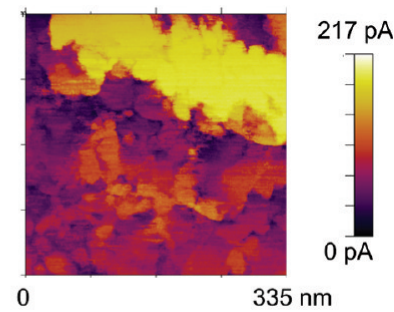

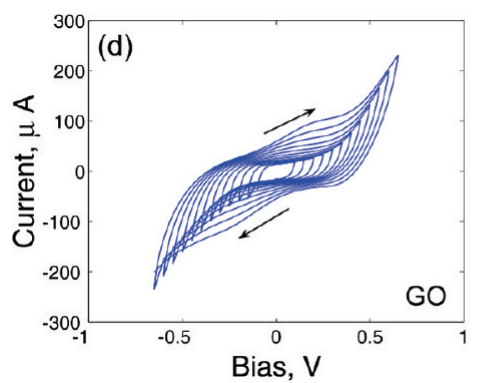
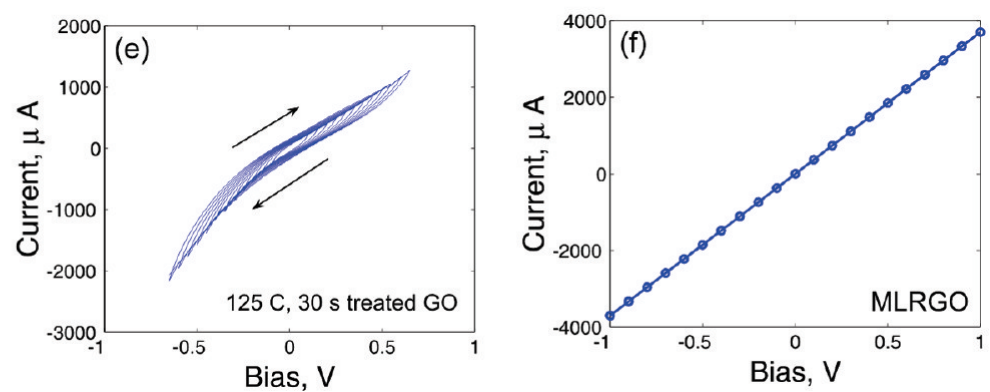

Figure 7. (a) Multilayer graphene oxide films, which have not been subject to thermal or electical reduction shows low surface conductivity as observed by tunneling current maps obtained with a Pt tip ( $\sim 10 \mathrm{mV}$ bias). (b) Upon partial reduction, conductive domains are observed in the tunneling current maps. (c) Tunneling current maps of chemically or thermally reduced multilayer graphene films show plateaus of current instead of conductive spots. (d) Cyclic current-voltage measurements are performed on two-terminal devices with unreduced graphene oxide. Hysteresis and changes in the IV curves show that graphene oxide films feature both resistive switching and capacitive charge storage. (e) Hysteresis is decreased for two-terminal devices fabricated using partially reduced graphene oxide films, and (f) no observable hysteresis is present in multilayer graphene films obtained by complete thermal reduction $\left(150^{\circ} \mathrm{C}\right.$, few minutes thermal exposure) of graphene oxide films.

reaction). Increased driving force $(\Delta G)$ greatly enhances the rate of the reaction, and it is possible to completely reduce graphene oxide in a few seconds. Also, for the oxidation case of partially reduced graphene oxide, the reaction is not spontaneous under ambient conditions and temperature. When graphene is positively charged, the oxidation is spontaneous. The reaction rate is observed to depend on initial oxygen coverage of graphene and is slower for graphene-like films. Both graphene quantum dots are formed and oxygen coverage change during the electrical reduction. The equilibrium size distribution of the quantum dots can be controlled with the applied voltage. In order to determine the equilibrium stoichiometry and structure, the partition function must be written for the graphene/graphene oxide system, also including the electrostatic energy due to the presence of excess charge.

Oxygen is known to bind to graphene in a number of different configurations, resulting in different band gaps and binding energies. ${ }^{23}$ The binding energy of individual oxygen atoms on graphene is also a function of coverage, as well as being dependent on the actual binding configuration (bond type) and presence of neighboring oxygen atoms. ${ }^{24} \mathrm{~A}$ thorough thermodynamic analysis of the phenomenon, as observed under ambient conditions, must take into account the myriad configurations of binding of oxygen, hydrogen, and hydroxide, in the presence of excess charge. Such an analysis must involve $a b$ initio calculations of total energy for a large number of configurations with a large number of atoms. We do not attempt to perform such an analysis in this study.

\section{CONCLUSIONS}

In summary, we demonstrate electrically induced reduction and oxidation of few-layer graphene films. If the repeatability and controllability of the observed effects can be improved, such electrochemical effects can be used in the design of novel micro- and nanoscale optoelectronic and memory devices. Demonstration of bias-controlled stoichiometry and nanoscale electronic structure can be viewed as electrically controlled formation of graphene quantum dots in graphene oxide. A similar quantum dot structure has been theoretically studied recently in a similar system (i.e., hydrogenated graphene). ${ }^{41}$ The results presented in this work suggest that electrical bias and charging effects are important in energetic analysis and design of such nanostructures. Observations suggest that the practical graphene material family of optoelectronic devices is potentially subject to nanoscale chemical modifications during operation. Graphene transistor experiments that are working under ambient conditions and with high voltages should take into account possible electrochemical effects. In a transistor structure, it could be possible to protect graphene from oxidation by proper capping. However, electrochemical reduction/oxidation reactions may still be present in the solid phase if the binding energy of oxygen on graphene is more than that in the capping (or gate) oxide material. Oxidation effects could be minimized 
by use of a dielectric that does not contain oxygen, such as $\mathrm{MgF}_{2}$. Charge-induced chemical reactions could also affect performance of graphene-based energy storage devices such as supercapacitors. The findings show the importance of electrochemical effects for graphene-based devices.

\section{METHODS}

Graphene oxide was synthesized from natural graphite (SP-1, Bay Carbon) by the Hummers method. ${ }^{36}$ Graphite oxide powder $(0.75 \mathrm{~g})$ was suspended in $50 \mathrm{~mL}$ of DI water and sonicated for $30 \mathrm{~min}$. The suspension was sequentially centrifuged at 8000 and $14500 \mathrm{rpm}$ for $30 \mathrm{~min}$ each, to completely eliminate unexfoliated graphene oxide particles.

Stable graphene suspensions were prepared by a fast and simple method. Hydrazine hydrate $(0.1 \mathrm{~mL}$, Merck) was added to the $10 \mathrm{~mL}$ of graphene oxide/water, and the mixture was heated to $85{ }^{\circ} \mathrm{C}$ for $1 \mathrm{~h}$. A few minutes after the addition of hydrazine hydrate, the color of the mixture became black. At the end of $1 \mathrm{~h}$, the graphene particles precipitated because of aggregation. Acetone (Sigma-Aldrich) was added to the solution with the same volume. After hydrazine treatment, the graphene surface contained hydrazone groups. ${ }^{37}$ Acetone could modify the surface graphene by reacting with hydrazone groups; therefore, addition of acetone dissolves graphene in the water and hydrazine hydrate mixture. To completely suspend aggregated graphene, $5 \mathrm{~min}$ of sonication was applied to the mixture.

Two-terminal devices were fabricated by sputtering $\mathrm{Au}-\mathrm{Pd}$ films on precleaned glass substrates by using a $300 \mu \mathrm{m}$ wide shadow mask. Graphene or GO suspensions were drop-casted on the devices fabricated. The films were dried under vacuum. Devices were characterized using a digital source meter (Keithley 2700) with a probe station. Graphene devices were annealed at $125-150^{\circ} \mathrm{C}$ for $30 \mathrm{~s}$ before measurements. Partially reduced graphene oxide samples were prepared by thermal annealing of graphene oxide films at $125^{\circ} \mathrm{C}$ for $30 \mathrm{~s}$. Thermal annealing processes were applied on a preheated hot plate. Electrochemical experiments were performed under ambient conditions in clean room conditions, at room temperature ( $T=25 \pm 0.5^{\circ} \mathrm{C}$ ) and $40 \pm 5 \%$ humidity as monitored by a humidity sensor, under 1 atm pressure.

Electrical and XPS (X-ray photoelectron spectroscopy) measurements were done on a Thermo K-Alpha system.

Multifrequency electrostatic force microscopy was implemented using modified commercial silicon cantilevers with spring constants of $2-4 \mathrm{~N} / \mathrm{m}$, and resonance frequencies of $65-75 \mathrm{kHz}$. The tips are double-stage electron-beam-induced deposited Pt tips with a tip radius of $2-5 \mathrm{~nm}$ as inspected by scanning electron microscopy. Low-frequency electrostatic excitation was applied at $5 \mathrm{kHz}$, and first and second time harmonics of the electrostatic forces were monitored using lock-in amplifiers (Stanford Research Systems, SR 830). The details of the highresolution multifrequency electrostatic force microscopy and spectroscopy can be found in references $38-40$.

Acknowledgment. This work was partially supported by TUBITAK under grant 107T547 and the State Planning Organization of Republic of Turkey, project UNAM. Authors thank M. Guler for critical reading of the manuscript, and S. Ciraci for discussions on graphene.

Supporting Information Available: Video micrographs, captured in bright-field transmitted light microscopy mode, demonstrating electrochromic effects, dentritic domain formation, and failure of two-terminal graphene oxide film devices upon application of various voltage pulses. This material is available free of charge via the Internet at http://pubs.acs.org.

\section{REFERENCES AND NOTES}

1. Novoselov, K. S.; Geim, A. K.; Morozov, S. V.; Jiang, D.; Katsnelson, M. I.; Grigorieva, I. V.; Dubonos, S. V.; Firsov, A. A. Two-Dimensional Gas of Massless Dirac Fermions in Graphene. Nature 2005, 438, 197-200.
2. Geim, A. K.; Novoselov, K. S. The Rise of Graphene. Nat. Mater. 2007, 6, 183-191.

3. Stankovich, S.; Dikin, D. A.; Dommett, G. H. B.; Kohlhaas, K. M.; Zimney, E. J.; Stach, E. A.; Piner, R. D.; Nguyen, S. T.; Ruoff, R. S. Graphene-Based Composite Materials. Nature 2006, 442, 282-286.

4. Meric, I.; Han, M. Y.; Young, A. F.; Ozyilmaz, B.; Kim, P.; Shepard, K. L. Current Saturation in Zero-Bandgap, TopGated Graphene Field-Effect Transistors. Nat. Nanotechnol. 2008, 3, 654-659.

5. Lin, Y. M.; Dimitrakopoulos, C.; Jenkins, K. A.; Farmer, D. B.; Chiu, H. Y.; Grill, A.; Avouris, P. 100-GHz Transistors from Wafer-Scale Epitaxial Graphene. Science 2010, 327, 662.

6. Novoselov, K. S.; Jiang, Z.; Zhang, Y.; Morozov, S. V.; Stormer, H. L.; Zeitler, U.; Maan, J. C.; Boebinger, G. S.; Kim, P.; Geim, A. K. Room-Temperature Quantum Hall Effect in Graphene. Science 2007, 315, 1379.

7. Schedin, F.; Geim, A. K.; Morozov, S. V.; Hill, E. W.; Blake, P.; Katsnelson, M. I.; Novoselov, K. S. Detection of Individual Gas Molecules Adsorbed on Graphene. Nat. Mater. 2007, 6, 652-655.

8. Wehling, T. O.; Novoselov, K. S.; Morozov, S. V.; Vdovin, E. E.; Katsnelson, M. I.; Geim, A. K.; Lichtenstein, A. I. Molecular Doping of Graphene. Nano Lett. 2008, 8, 173-177.

9. Robinson, J. T.; Perkins, F. K.; Snow, E. S.; Wei, Z.; Sheehan, P. E. Reduced Graphene Oxide Molecular Sensors. Nano Lett. 2008, 8, 3137-3140.

10. Stoller, M. D.; Park, S.; Zhu, Y.; An, J.; Ruoff, R. S. GrapheneBased Ultracapacitors. Nano Lett. 2008, 8, 3498-3502.

11. Yoo, E.; Kim, J.; Hosono, E.; Zhou, H.; Kudo, T.; Honma, I. Large Reversible Li Storage of Graphene Nanosheet Families for Use in Rechargeable Lithium Ion Batteries. Nano Lett. 2008, 8, 2277-2282.

12. Wang, L.; Lee, K.; Sun, Y.; Lucking, M.; Chen, Z.; Zhao, J. J.; Zhang, S. B. Graphene Oxide as an Ideal Substrate for Hydrogen Storage. ACS Nano 2009, 3, 2995-3000.

13. Mueller, T.; Xia, F.; Avouris, P. Graphene Photodetectors for High-Speed Optical Communications. Nat. Photonics 2010, 4, 297-301.

14. Gokus, T.; Nair, R. R.; Bonetti, A.; Bohmler, M.; Lombardo, A.; Novoselov, K. S.; Geim, A. K.; Ferrari, A. C.; Hartschuh, A. Making Graphene Luminescent by Oxygen Plasma Treatment. ACS Nano 2009, 3, 3963-3968.

15. Sun, Z.; Hasan, T.; Torrisi, F.; Popa, D.; Privitera, G.; Wang, F.; Bonaccorso, F.; Basko, D. M.; Ferrari, A. C. Graphene ModeLocked Ultrafast Laser. ACS Nano 2010, 4, 803-810.

16. Bolotina, K. I.; Sikesb, K. J.; Jianga, Z.; Klimac, M.; Fudenberga, G.; Honec, J.; Kima, P.; Stormera, H. L. Ultrahigh Electron Mobility in Suspended Graphene. Solid State Commun. 2008, 146, 351-355.

17. Sevincli, H.; Topsakal, M.; Ciraci Superlattice, S. Structures of Graphene-Based Armchair Nanoribbons. Phys. Rev. B 2008, 78, 245402.

18. Han, M. Y.; Ozyilmaz, B.; Zhang, Y.; Kim, P. Energy BandGap Engineering of Graphene Nanoribbons. Phys. Rev. Lett. 2007, 98, 206805.

19. Bai, J.; Zhong, X.; Jiang, S.; Huang, Y.; Duan, X. Graphene Nanomesh. Nat. Nanotechnol. 2010, 5, 190-194.

20. Li, Y.; Sinitskii, A.; Tour, J. M. Electronic Two-Terminal Bistable Graphitic Memories. Nat. Mater. 2008, 7, 966971.

21. He, C. L.; Zhuge, F.; Zhou, X. F.; Li, M.; Zhou, G. C.; Liu, Y. W.; Wang, J. Z.; Chen, B.; Su, W. J.; et al. Nonvolatile Resistive Switching in Graphene Oxide Thin Films. Appl. Phys. Lett. 2009, 95, 232101.

22. Echtermeyer, T. J.; Lemme, M. C.; Baus, M.; Szafranek, B. N.; Geim, A. K.; Kurz, H. Nonvolatile Switching in Graphene 
Field-Effect Devices. IEEE Electron Device Lett. 2008, 29, 952-954.

23. Yan, J.; Xian, L.; Chou, M. Y. Structural and Electronic Properties of Oxidized Graphene. Phys. Rev. Lett. 2009, 103, 086802.

24. Boukhvalov, D. W.; Katsnelson, M. I. Modeling of Graphite Oxide. J. Am. Chem. Soc. 2008, 130, 10697-10701.

25. Jung, I.; Dikin, D. A.; Piner, R. D.; Ruoff, R. S. Tunable Electrical Conductivity of Individual Graphene Oxide Sheets Reduced at Low Temperatures. Nano Lett. 2008, 8, 4283-4287.

26. Lopez, V.; Sundaram, R. S.; Gomez-Navarro, C.; Olea, D.; Burghard, M.; Gomez-Herrero, J.; Zamora, F.; Kern, K. Chemical Vapor Deposition Repair of Graphene Oxide: A Route to Highly-Conductive Graphene Monolayers. Adv. Mater. 2009, 21, 1-4.

27. Cote, L. J.; Cruz-Silva, R.; Huang, J. Flash Reduction and Patterning of Graphite Oxide and Its Polymer Composite. J. Am. Chem. Soc. 2009, 131, 11027-11032.

28. Gomez-Navarro, C.; Thomas Weitz, R.; Bittner, A. M.; Scolari, M.; Mews, A.; Burghard, M.; Kern, K. Electronic Transport Properties of Individual Chemically Reduced Graphene Oxide Sheets. Nano Lett. 2007, 7, 3499-3503.

29. Wei, Z.; Wang, D.; Kim, S.; Kim, S.; Hu, Y.; Yakes, M. K.; Laracuente, A. R.; Dai, Z.; Marder, S. R.; et al. Nanoscale Tunable Reduction of Graphene Oxide for Graphene Electronics. Science 2010, 328, 1373.

30. Sessi, P.; Guest, J. R.; Bode, M.; Guisinger, N. P. Patterning Graphene at the Nanometer Scale via Hydrogen Desorption. Nano Lett. 2009, 9, 4343-4347.

31. Mativetsky, J. M.; Treossi, E.; Orgiu, E.; Melucci, M.; Veronese, G. P.; Samori, P.; Palermo, V. Local Current Mapping and Patterning of Reduced Graphene Oxide. J. Am. Chem. Soc. 2010, 132, 14130-14136.

32. Nair, R. R.; Blake, P.; Grigorenko, A. N.; Novoselov, K. S.; Booth, T. J.; Stauber, T.; Peres, N. M. R.; Geim, A. K. Fine Structure Constant Defines Visual Transparency of Graphene. Science 2008, 320, 1308.

33. Avouris, P.; Martel, R.; Hertel, T.; Sandstrom, R. AFM-TipInduced and Current-Induced Local Oxidation of Silicon and Metals. Appl. Phys. A: Mater. Sci. Process. 1998, 66, S659-S667.

34. Okada, Y.; Amano, S.; Kawabe, M.; Harris, J. S. Basic Mechanisms of an Atomic Force Microscope Tip-Induced Nano-oxidation Process of GaAs. J. Appl. Phys. 1998, 83, 7998-8001.

35. Avouris, P.; Hertel, T.; Martel, R. Atomic Force Microscope Tip-Induced Local Oxidation of Silicon: Kinetics, Mechanism and Nanofabrication. Appl. Phys. Lett. 1997, 71, 285287.

36. Hummers, W. S.; Offeman, R. E. Preparation of Graphitic Oxide. J. Am. Chem. Soc. 1958, 80, 1339.

37. Stankovich, S.; Piner, R. D.; Chen, X.; Wu, N.; Nguyen, S. T.; Ruoff, R. S. Stable Aqueous Dispersions of Graphitic Nanoplatelets via the Reduction of Exfoliated Graphite Oxide in the Presence of Poly(sodium 4-styrenesulfonate). J. Mater. Chem. 2006, 16, 155-158.

38. Ding, X. D.; An, J.; Xu, J. B.; Li, C.; Zeng, R. Y. Improving Lateral Resolution of Electrostatic Force Microscopy by Multifrequency Method under Ambient Conditions. Appl. Phys. Lett. 2009, 94, 223109.

39. Ziegler, D.; Reichen, J.; Naujoks, N.; Stemmer, A. Compensating Electrostatic Forces by Single-Scan Kelvin Probe Force Microscopy. Nanotechnology 2007, 18, 225505.

40. Bostanci, U.; Abak, M. K.; Aktaş, O.; Dâna, A Nanoscale Charging Hysteresis Measurement by Multifrequency Electrostatic Force Spectroscopy. Appl. Phys. Lett. 2008, 92, 093108.

41. Singh, A. K.; Penev, E. S.; Yakobson, B. I. Vacancy Clusters in Graphane as Quantum Dots. ACS Nano 2010, 4, 35103514.

42. Singh, A. K.; Yakobson, B. I. Electronics and Magnetism of Patterned Graphene Nanoroads. Nano Lett. 2009, 9, 1540-1543. 